\title{
CALCULO DE TIEMPOS T1 Y T2 IN VITRO
}

\section{Dr. Marcelo Gálvez M(1), T.M. Mauricio Farías $A^{(1)}$, PhD Takeshi Asahi $K^{(1)}$, Eduardo Bravo $C^{(1)}$.}

1. Servicio de Neurorradiología Instituto de Neurocirugía Dr. Asenjo.

Abstract: The present work deals with the standardization of the parameters of different Magnetic Resonance sequences, in order to quantify the values of the relaxation times $T_{1}$ y $T_{2}$ from the obtained set of images.

In vitro images from a $1.5 T$ MR scanner were experimentally obtained for different biological tissues corresponding to muscle, fat and water. From this data the $T_{1}$ and $T_{2}$ curves were calculated for the different tissues. The sequences correspond to spin-echo by varying $T R$ for $T_{1}$, and turbo spin-echo by varying $T E$ for $T_{2}$. Finally, the parameters $T_{1}$ and $T_{2}$ of the corresponding curves of longitudinal and transversal relaxation were obtained by means of the fitting with the exponential theoretical curves. The experimental $T_{1}$ values correspond to $951 \mathrm{~ms}$ for the muscles, 238 ms for the fat and $2813 \mathrm{~ms}$ for the water. The experimental $T_{2}$ values correspond to $71 \mathrm{~ms}$ for the muscles, $81 \mathrm{~ms}$ for the fat and $704 \mathrm{~ms}$ for the water. These studies show the feasibility of calculating this parameter, in order to be utilized for different quantitative analysis of the images for magnetic resonance.

Keywords: Echo times, Miliseconds, $T_{1}$ relaxation times, $T_{2}$ relaxation times, Repetition times, $R M$ signal.

Resumen: El objetivo del trabajo consiste en cuantificar los valores de los tiempos de relajación $T_{\text {, }}$ y $\mathrm{T}_{2}$ a través de una simple modificación de las secuencias convencionales.

En forma experimental se obtuvieron imágenes in vitro en un resonador magnético de $1.5 T$ de diferentes tejidos biológicos correspondientes a músculos, lípidos y agua, a partir de las cuales se obtuvieron las respectivas curvas $T_{1}$ y $T_{2}$. Las secuencias utilizadas corresponden a espín-eco para las curvas $T_{1}$ mediante la variación del TR y turbo espín-eco para las curvas $T_{2}$, por medio de la variación del TE.

Gálvez M. Cálculo de tiempos T1 y T2 In Vitro. Rev Chil Radiol 2005; 11: 109-115.

Correspondencia: Dr. Marcelo Gálvez M.

José Miguel Infante 553, Providencia, Santiago de Chile. Fono: 2003371-2003372200 3288

www.neurorradiologia.cl
Finalmente los parámetros $T_{1}$ y $T_{2}$ de las respectivas curvas de relajación longitudinal y transversal se obtuvieron mediante el ajuste con las curvas exponenciales teóricas. Los valores $T_{1}$ resultantes fueron $951 \mathrm{~ms}$ para el músculo, $238 \mathrm{~ms}$ para los lípidos y $2813 \mathrm{~ms}$ para el agua. Los valores $\mathrm{T}_{2}$ resultantes fueron $71 \mathrm{~ms}$ para el músculo, $81 \mathrm{~ms}$ para los lípidos y $704 \mathrm{~ms}$ para el agua. Lo anterior demuestra la factibilidad de calcular estos parámetros, con el objetivo de ser utilizados en los diferentes análisis cuantitativos de las imágenes por resonancia magnética.

Palabras clave: Señal de RM, TE, Tiempo $T_{1}$, Tiempo $T_{2} T R$.

Señal de RM: Señal leída luego de la excitación que nos entrega información proveniente del retorno de la magnetización longitudinal y la pérdida de la magnetización transversa.

TE: Tiempo comprendido entre el impulso de excitación activación y el eco.

Tiempo $\mathrm{T}_{1}$ : Constante de tiempo especifica del tejido que describe el retorno de la magnetización longitudinal al estado de equilibrio (63\% de su valor máximo).

Tiempo $\mathrm{T}_{2}$ : Constante de tiempo especifica del tejido que describe la pérdida de la magnetización transversa ( $37 \%$ de su valor máximo).

TR: Tiempo comprendido entre dos pulsos de excitación.

\section{Objetivos}

Describir una metodología para la confección de las curvas de relajación longitudinal y relajación transversal.

Determinar los valores $T_{1}$ y $T_{2}$ del agua, grasa (lípidos) y músculo en una muestra in vitro.

\section{Introducción}

La señal de resonancia magnética (RM) convencional proviene de los núcleos de los átomos de hidrógeno y es modulada por parámetros extrínsecos como lo es el campo magnético e intrínsecos como lo constituyen las características propias de los tejidos.

Entre los mecanismos de contraste intrínseco comúnmente medidos en RM encontramos el tiempo 
de relajación $T_{1}$, el tiempo de relajación $T_{2}$ y la densidad protónica (DP). Esta ponderación es determinada por la selección de parámetros de tiempo de la secuencia de pulso que se usa en cada muestreo.

Entre las secuencias de pulso comúnmente utilizadas están las secuencias espín-eco (SE), en las cuales podemos identificar el Tiempo de Repetición (TR) y el Tiempo de Eco (TE). El TR controla la cantidad de ponderación $T_{1}$, mientras que el TE controla la cantidad de ponderación $\mathrm{T}_{2}$. Si deseamos obtener imágenes ponderadas en contraste $T_{1}$ entonces debemos seleccionar TR cortos que están en valores de 400-550 milisegundos (ms) y TE cortos generalmente menores de $25 \mathrm{~ms}$. Si por el contrario queremos lograr contraste o ponderación $\mathrm{T}_{2}$ debemos seleccionar TR más largos en valores de 2000 ms o más y TE altos en valores de 80-120 ms. Para obtener una ponderación DP debemos disminuir el efecto $T_{1}$ subiendo los tiempos de TR sobre 2000-2500 ms y disminuir el efecto $T_{2}$ bajando el TE a 25 ms o menos.

\section{Curvas y Tiempo T1}

También llamado spin-lattice o tiempo de relajación longitudinal. Corresponde a un tiempo constante tejido-específico para los protones y es una medición del tiempo que se requiere para lograr una realineación con el campo magnético externo. La constante $\mathrm{T} 1$ nos informa cuan rápido el movimiento espín del núcleo puede emitir la energía de radiofrecuencia absorbida al medio circundante, por lo que se entiende la dependencia de factores como tipo de moléculas, movilidad y entorno(1).

Tal como se describe el comportamiento de varios procesos naturales como el aumento bacterial - la caída de la radiactividad, la relajación longitudinal tiene la forma de una exponencial creciente regulada por una constante de tiempo llamada $T_{1}(\mathrm{~ms})$, que caracteriza su recuperación. Matemáticamente se expresa por la siguiente fórmula que llamaremos ecuación $(A)$ y que describe una curva creciente con un valor máximo $D_{p}$ (Gráfico 1$)$.

$$
M_{T_{1}}(t)=D p\left(1-k^{-t / T_{1}}\right)
$$

De esto concluimos que si $k=1$, cuando el tiempo transcurrido a partir del pulso inicial (t) sea igual a $T_{1}, M_{T 1} / D_{p}$ será igual a 1- $\exp (-1)=1-0,37=$ 0,63 , lo que quiere decir que el $T_{1}$ es el tiempo que tarda la magnetización en recuperar un $63 \%$ de su valor.

\section{Curvas y Tiempo T2}

La recuperación de la magnetización longitudinal es acompañada por el decaimiento de la magnetización transversal caracterizada por la

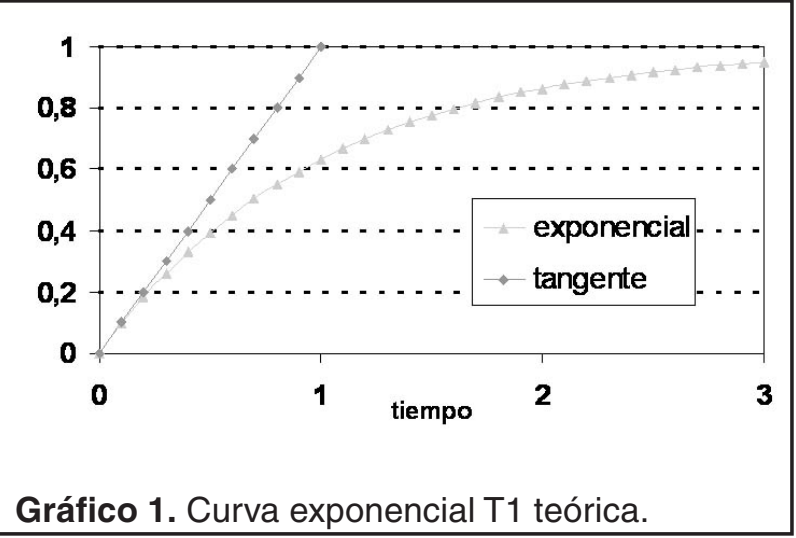

constante $T_{2}$. Recibe el nombre de tiempo de relajación espín-espín o tiempo de relajación transversal(3).

Estos dos procesos se producen en forma independiente. Los fenómenos de relajación espínespín afectan a la duración natural de la señal de inducción libre, durante la cual los diversos componentes de magnetización en el plano $x y$ se mantienen más o menos en fase. Cuando acaba el pulso de excitación, los núcleos no sólo perciben el campo estático externo sino también los campos locales asociados con las propiedades magnéticas de los núcleos vecinos, de forma que van adquiriendo una frecuencia de precesión ligeramente diferente, lo que provoca una caída de la magnetización transversal. Si el campo magnético estático fuese perfectamente uniforme, bastaría medir la velocidad de amortiguación en la señal de inducción libre para determinar el valor de $T_{2}$. No obstante, los campos generados por imanes reales casi nunca son perfectos. Hasta las sutilísimas imperfecciones de los mejores imanes utilizados para espectroscopía de RM hacen que la señal de inducción libre decaiga con mayor rapidez de lo que acontecería en presencia de un campo magnético perfectamente homogéneo. La constate de tiempo que define la velocidad real de la caída de la señal de un campo imperfecto se designa por $\mathrm{T}_{2}{ }^{*}$ para distinguirla del verdadero tiempo de relajación $T_{2}$.

A pesar de todo ello, podemos determinar el valor intrínseco de $\mathrm{T}_{2}$ para una muestra aunque esté sometida a un campo imperfecto, ya que las desigualdades del campo magnético son constantes, lo que permite identificarlas y cancelarlas. Se recoge la señal en forma de «eco de espín» o de una serie de ecos, aplicando un perfil especial de pulso de radiofrecuencias, conocido como la secuencia de pulsos Carr-Purcell modificada ${ }^{(4)}$. En dicha secuencia, la señal inicial de amortiguación de inducción libre y cada uno de los ecos de espín individual decaen con una constante de tiempo $\mathrm{T}_{2}{ }^{*}$, aunque las alturas del pico de los sucesivos ecos de espín decaen con una 
constante de tiempo igual al valor intrínseco de $T_{2}$ en la muestra.

La evolución de la magnetización transversal con el tiempo hasta que se anula corresponde a una sinusoide a la frecuencia de relajación hasta que se anula, amortiguada por una exponencial decreciente. Se llama $\mathrm{T}_{2}{ }^{*}$ al fenómeno que considera los factores que influyen en el desfase de los espínes $0 T_{2}$ si no se consideran ni variaciones locales ni la influencia de las heterogeneidades del campo magnético externo y sólo atiende a la composición y estructuración propia del tejido.

La envolvente exponencial que regula el decrecimiento se expresa en la siguiente fórmula que llamaremos ecuación B y que describe una curva exponencial decreciente, como se muestra en el gráfico 2.

$$
M_{T 2}(t)=M_{0 T 2} e^{-t / T 2}
$$

Cuando $t=T_{2}, M_{\text {T2 }}(t) / M_{\text {от2 }}$ es igual a 0.37 lo que equivale a que el $T_{2}$ es el tiempo que tiene que transcurrir para que la magnetización transversal pierda el $63 \%$ de su valor.

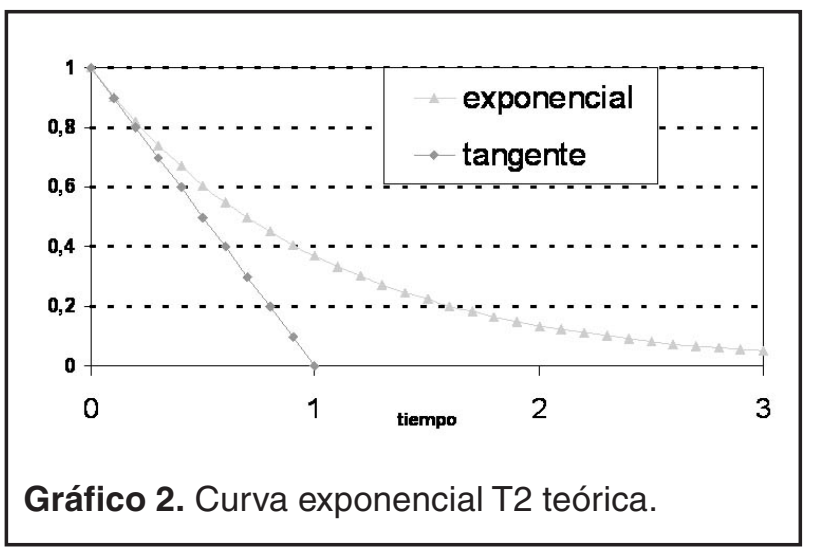

\section{Material y métodos}

Se analizo una muestra constituida a partir de una porción de pared costal de porcino, en la cual estudiamos tres tejidos diferentes definidos a priori como representativos de ella. Así identificamos grasa y músculos inmersos en agua contenidos en un recipiente de $1.500 \mathrm{cc}$ de capacidad (Figura 1).

La experiencia se realizó en un resonador magnético de $1.5 \mathrm{~T}$, de tipo superconductivo, de amplitud máxima de gradiente de $66 \mathrm{mT} / \mathrm{m}$ (Figura 2). En la obtención de los datos se utilizó una antena de polarización circular (cuadratura) en cuyo centro se depositó la muestra. Todas las medidas se realizaron en el isocentro del imán con los mismos parámetros de: orientación de corte coronal, FOV

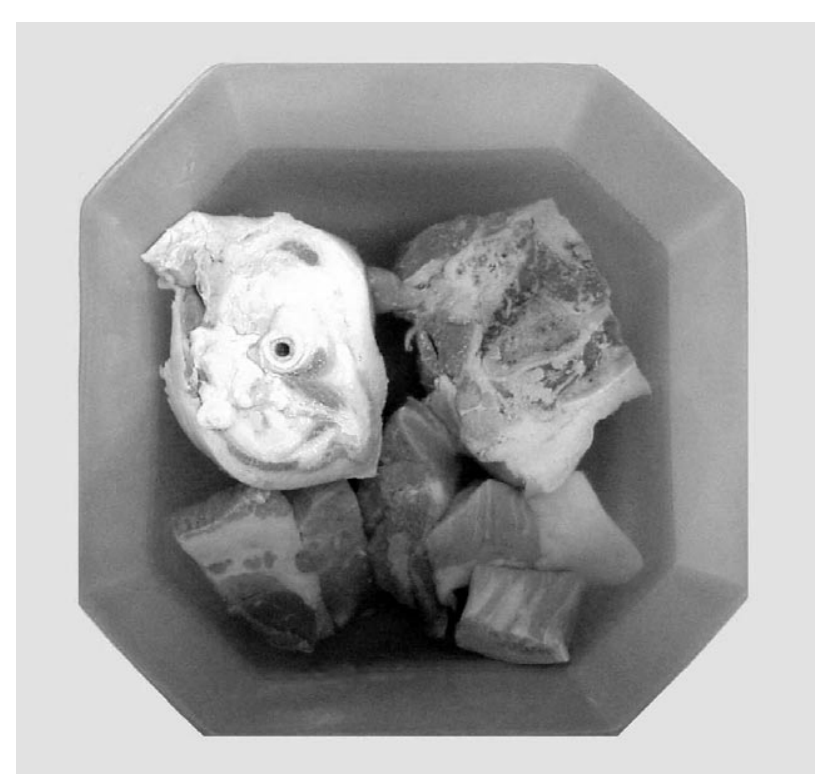

Figura 1. Muestras constituidas por carne porcina utilizada en nuestra medición.

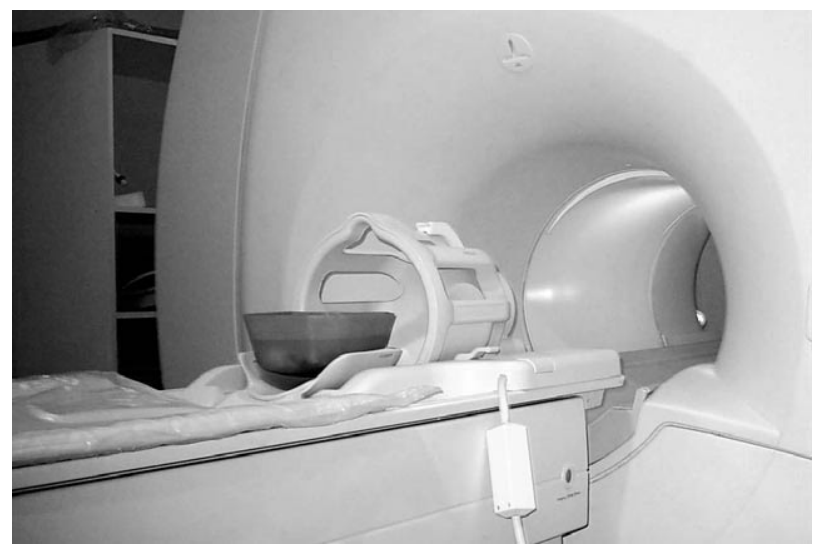

Figura 2. Instalación de la muestra en el resonador.

$200 \mathrm{~mm}$, matriz de 256x256, espesor de corte de 5 $\mathrm{mm}$, ancho de banda de $130 \mathrm{~Hz} /$ píxel.

Para la obtención de los datos de la curva $T_{1}$ se variaron los valores de TR en forma creciente en $28 \mathrm{~ms}, 40 \mathrm{~ms}, 100 \mathrm{~ms}, 300 \mathrm{~ms}, 500 \mathrm{~ms}, 1.000 \mathrm{~ms}$, $2.000 \mathrm{~ms}, 3.000 \mathrm{~ms}$ y $4.200 \mathrm{~ms}$ manteniendo constante además en este caso en tiempo TE en 15 ms. Algunas de las imágenes obtenidas se ilustran en la figura 3.

Por otro lado se lograron datos de la curva $\mathrm{T}_{2}$. La variación de los valores de TE se efectuaron en forma creciente, según lo permitió el sistema, de 65 $\mathrm{ms}, 78 \mathrm{~ms}, 91 \mathrm{~ms}, 104 \mathrm{~ms}, 117 \mathrm{~ms}, 169 \mathrm{~ms}, 253 \mathrm{~ms}$, $506 \mathrm{~ms}, 658 \mathrm{~ms}, 1.010 \mathrm{~ms}, 1.240 \mathrm{~ms}, 1.670 \mathrm{~ms}$ y $2510 \mathrm{~ms}$ manteniendo constante en este caso el valor de TR en $4.000 \mathrm{~ms}$. Algunas de las imágenes obtenidas se ilustran en la figura 4 . 

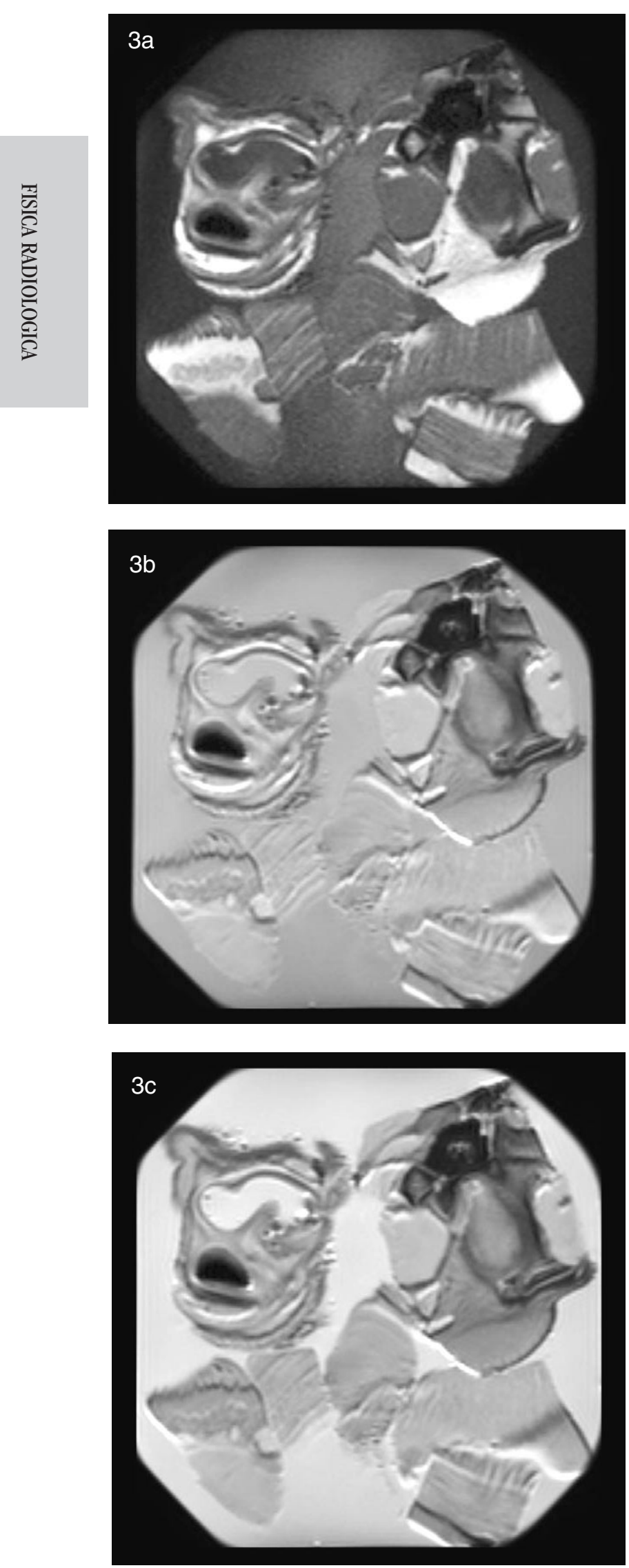

Figura 3 a-c. Imágenes por RM obtenidas sobre la muestra manteniendo TE fijo en $15 \mathrm{~ms}$ y variando TR. a) $100 \mathrm{~ms}$. b) $2.000 \mathrm{~ms}$. c) $4.200 \mathrm{~ms}$.
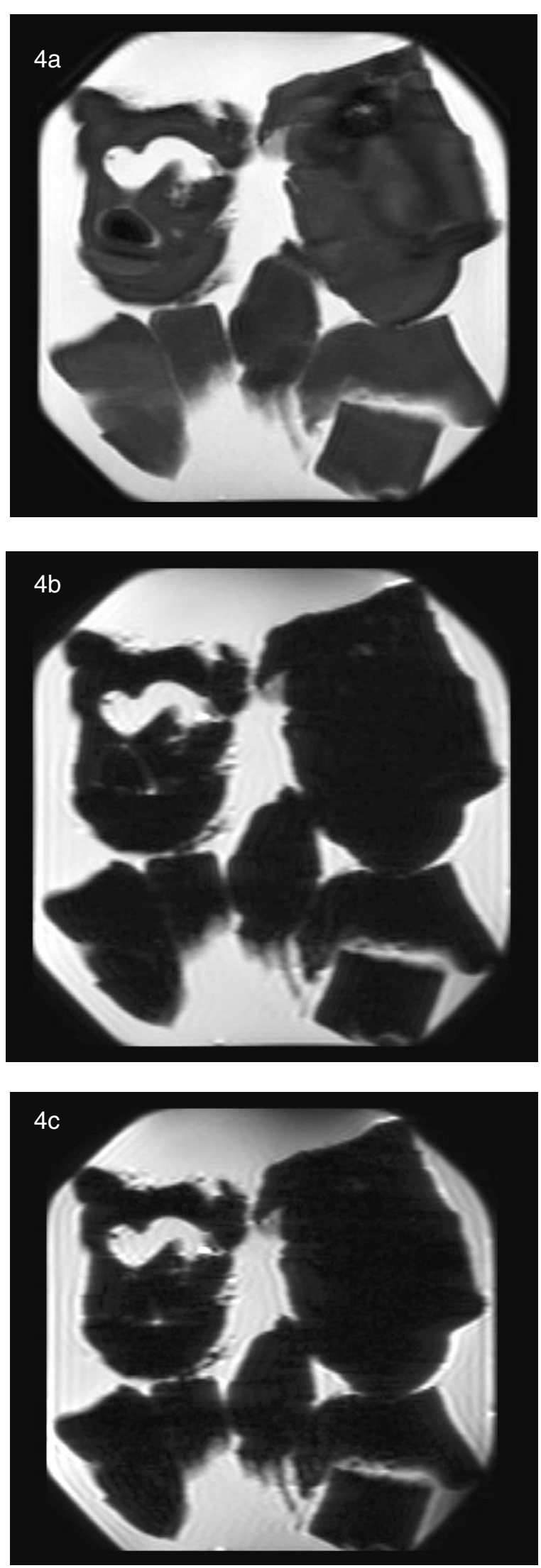

Figura 4 a-c. Imágenes por RM obtenidas sobre la muestra manteniendo TR fijo en 4000 ms y variando TE. a) 104 ms. b) $1.010 \mathrm{~ms}$. c) $2.510 \mathrm{~ms}$. 
Una vez obtenidos los datos se identificaron las fases de agua, grasa, y músculo y sobre ellas se realizaron mediciones de intensidad de señal a través de un ROI constante para todas $\left(110 \mathrm{~mm}^{2}\right)$ las medidas. Los resultados serán registrados según:

$$
I_{s}=A\left[N_{0}\right]\left(1-e^{-T R / T 1}\right) e^{-T E / T 2}
$$

donde $\mathrm{I}_{s}$ es la intensidad de señal, $A$ es una constante de proporcionalidad, y $\mathrm{N}_{0}$ es la densidad de protones. A partir de los datos obtenidos en forma experimental de las curvas $T_{1}$ y $T_{2}$ del músculo, grasa y agua, es posible calcular los tiempos característicos de cada uno de estos elementos.

El procedimiento utilizado corresponde a la minimización del error cuadrado entre la curva exponencial ideal y los datos experimentales. Como antes fue mencionado, los parámetros de la curva $T_{1}$ son el valor máximo $D_{p}$ y el tiempo característico $\tau_{1}$ (tau). Para el caso de la curva $T_{2}$, sus parámetros son el valor inicial $\mathrm{M}_{\text {от2 }}$ y el tiempo característico $\tau_{2}$. El sistema de dos ecuaciones a resolver es no lineal, por lo que se minimizó el primero de los parámetros manteniendo el segundo fijo. Se repitió este proceso de manera alternada, variando el segundo parámetro y manteniendo el primero fijo.

\section{Resultados y discusión}

Para músculo, lípido y agua se calcularon los tiempos $T_{1} y T_{2}$. Sin embargo, para el agua solamente no se pudo obtener un buen ajuste al calcular su $T_{1}$, debido a que se necesita tener la intensidad de señal con un TR superior al utilizado en nuestro experimento. En la figura 5 se puede apreciar imágenes con ponderaciones estándar $\mathrm{T}_{1}, \mathrm{~T}_{2} \mathrm{y}$ Densidad Protónica.

Se graficó la intensidad de señal en función del TR (Gráfico 3). Para los ajustes de las curvas teóricas, se utilizó la fórmula de la ecuación (A) y los resultados obtenidos se muestran en la Tabla 1.

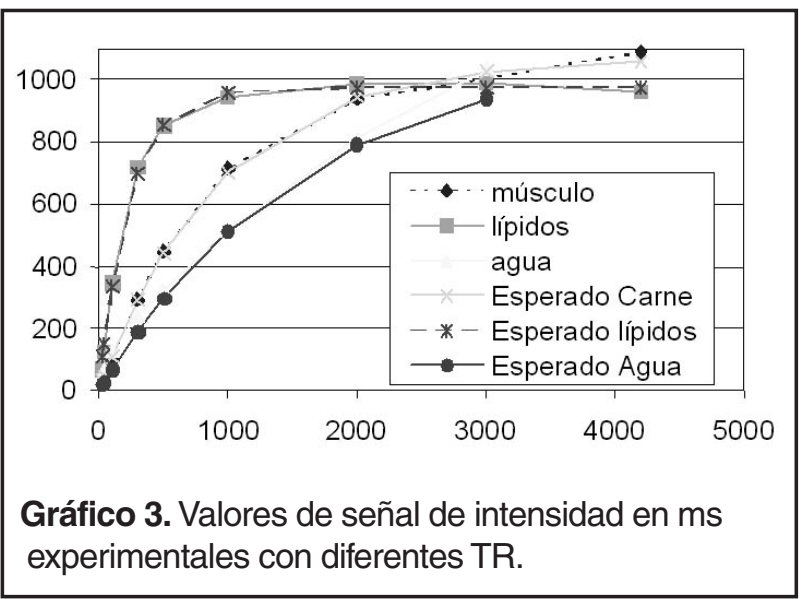

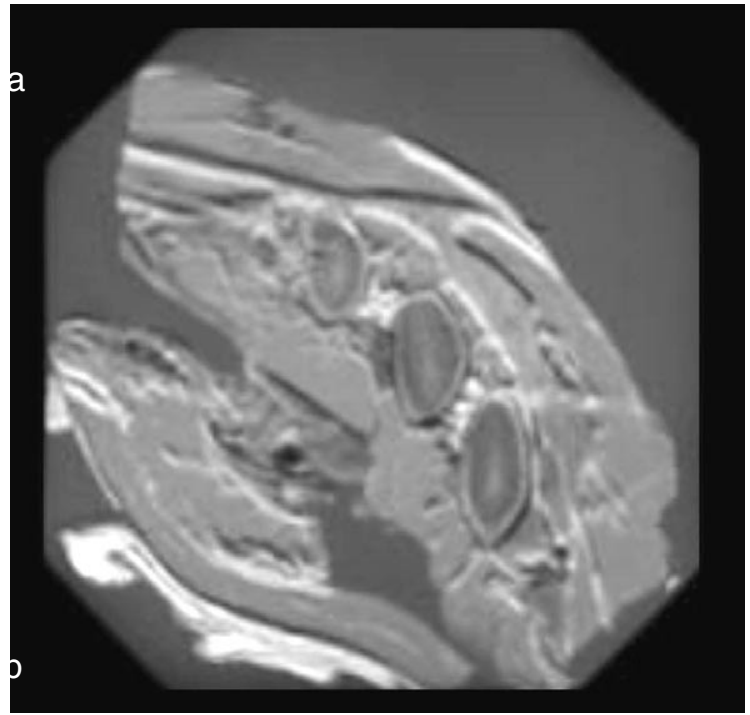
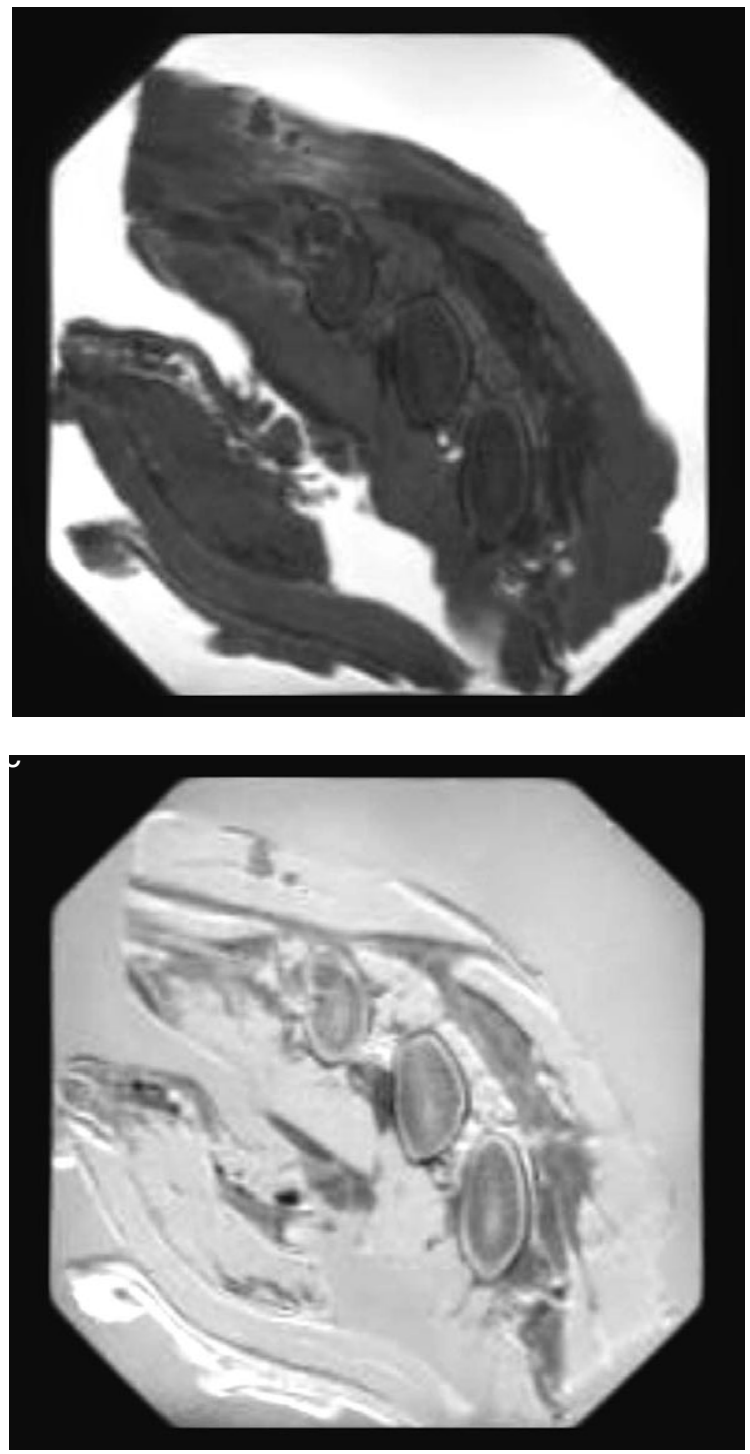

Figura 5 a-c. Imágenes por RM obtenidas de una muestra constituida por carne porcina, utilizando los parámetros de contraste clásicos (T1; T2; DP). a) Imagen T1 con $T R=500 \mathrm{~ms}$ y TE=15 ms. b) Imagen $T 2$ con $T R=300 \mathrm{~ms} y$ $T E=120 \mathrm{~ms}$. c) Imagen DP con $T R=1.500 \mathrm{~ms}$ y $T R=20 \mathrm{~ms}$. 
Tabla I. Valores de intensidad de señal en ms de 3 tejidos biológicos con ROI fijo y TE fijo (15 ms).

\begin{tabular}{|c|c|c|c|c|c|c|c|c|c|}
\hline \multicolumn{4}{|c|}{ TE FIJO $15 \mathrm{~ms}$} & \multicolumn{3}{|c|}{ Valor esperado } & \multicolumn{3}{|c|}{ ErrCuad } \\
\hline TR & Músculo & Lípidos & Agua & Músculo & Lípidos & Agua & Músculo & Lípidos & Agua \\
\hline 28 & 17 & 68 & 40 & 31 & 108 & 19 & 207,0 & $1.615,6$ & 441,0 \\
\hline 40 & 33 & 123 & 67 & 45 & 151 & 27 & 133,5 & 773,3 & $1.596,6$ \\
\hline 100 & 98 & 346 & 127 & 108 & 334 & 66 & 98,9 & 133,6 & $3.574,1$ \\
\hline 300 & 292 & 718 & 242 & 292 & 699 & 188 & 0,1 & 377,4 & $2.962,8$ \\
\hline 500 & 445 & 852 & 321 & 441 & 856 & 295 & 12,5 & 14,3 & 686,8 \\
\hline 1.000 & 714 & 944 & 510 & 701 & 961 & 512 & 163,4 & 274,7 & 3,5 \\
\hline 2.000 & 937 & 986 & 811 & 944 & 975 & 789 & 48,5 & 121,4 & 463,1 \\
\hline 3.000 & 1.004 & 990 & 1.033 & 1.028 & 975 & 940 & 576,2 & 219,1 & $8.644,5$ \\
\hline \multirow[t]{3}{*}{4.200} & 1.090 & 960 & 1.350 & 1.060 & 975 & - & 897,6 & 231,0 & - \\
\hline & & Max & & $1.072,5$ & 975,2 & $1.118,4$ & $2.137,7$ & $3.760,5$ & $18.472,6$ \\
\hline & & Tau & & 942,7 & 238,1 & $1.634,2$ & & & \\
\hline
\end{tabular}

Las curvas de intensidad de señal en función de TE se ilustran en el gráfico 4. Las curvas teóricas fueron ajustadas con la ecuación (B) minimizando el error cuadrático medio, y los resultados obtenidos se muestran en la Tabla 2.

Los resultados experimentales obtenidos de la gráfica $T_{1}$ se encuentran detallados en la Tabla 3 , junto a ellos los valores de referencia encontrados en la literatura(6). Del mismo modo se presentan los cálculos realizados a partir de la gráfica $T_{2}$ en la Tabla 4.

De la realización de este muestreo podemos confirmar que diferentes tipos de tejidos tienen diferentes tiempos de relajación, lo que se puede estandarizar a través de sus constantes $T_{1}$ y $T_{2}$.

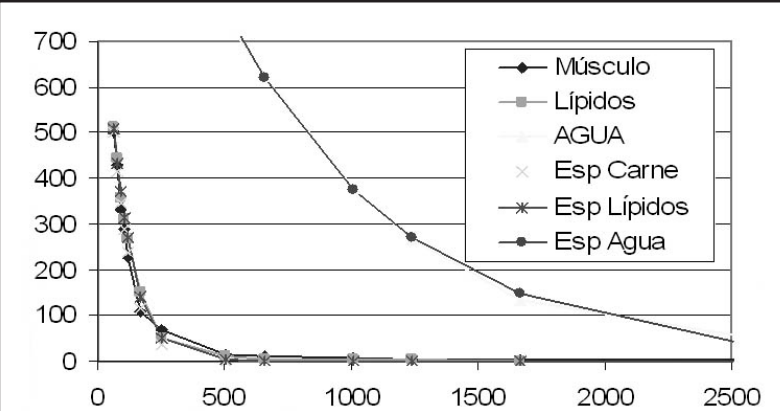

Gráfico 4. Valores de señal de intensidad en ms experimentales con diferentes TE.

Tabla II. Valores de intensidad de señal en ms de 3 tejidos biológicos con ROI fijo y TR fijo (4000 ms).

\begin{tabular}{|c|c|c|c|c|c|c|c|c|c|}
\hline \multirow[b]{2}{*}{ TE } & \multicolumn{3}{|c|}{ TR $4000 \mathrm{~ms}$} & \multicolumn{3}{|c|}{ Valor esperado } & \multicolumn{3}{|c|}{ ErrCuad } \\
\hline & Músculo & Lípidos & Agua & Músculo & Lípidos & Agua & Músculos & Lípidos & Agua \\
\hline 65 & 502 & 511 & 1.403 & 414 & 435 & 1.393 & $7.791,8$ & $5.826,5$ & 99,1 \\
\hline 78 & 429 & 443 & 1.395 & 373 & 393 & 1.370 & $3.172,9$ & $2.489,9$ & 628,3 \\
\hline 91 & 333 & 359 & 1.373 & 336 & 356 & 1.347 & 7,2 & 12,2 & 665,3 \\
\hline 104 & 290 & 311 & 1.369 & 302 & 322 & 1.325 & 153,1 & 110,5 & $1.948,9$ \\
\hline 117 & 228 & 267 & 1.355 & 272 & 291 & 1.303 & $1.968,5$ & 564,8 & $2.717,2$ \\
\hline 169 & 110 & 152 & 1.315 & 179 & 195 & 1.219 & $4.803,5$ & $1.806,6$ & $9.305,0$ \\
\hline 253 & 70 & 51 & 1.076 & 91 & 102 & 1.094 & 452,2 & $2.559,6$ & 312,6 \\
\hline 506 & 15 & 11 & 750 & 12 & 14 & 790 & 9,4 & 11,3 & $1.579,6$ \\
\hline 658 & 10 & 5 & 626 & 4 & 4 & 649 & 42,0 & 0,3 & 549,0 \\
\hline 1.010 & 6 & 2 & 379 & 0 & 0 & 413 & 33,6 & 2,9 & $1.146,1$ \\
\hline 1.240 & 5 & 3 & 267 & 0 & 0 & 307 & 24,7 & 8,7 & $1.606,1$ \\
\hline 1.670 & 5 & 1 & 132 & 0 & 0 & 177 & 25,0 & 1,0 & $1.986,4$ \\
\hline \multirow[t]{2}{*}{2.510} & 4 & 1 & 56 & 0 & 0 & 60 & 16,0 & 1,0 & 15,2 \\
\hline & & & $\begin{array}{r}\text { Max } \\
\text { Tau }\end{array}$ & $\begin{array}{r}1.253,6 \\
70,7\end{array}$ & $\begin{array}{r}1.127,3 \\
81,7\end{array}$ & $\begin{array}{r}1.577,3 \\
703,3\end{array}$ & $18.500,0$ & $13.395,3$ & $16.500,0$ \\
\hline
\end{tabular}


Tabla III. Valores T1 obtenidos a partir de la fórmula de Carr-Purcell (A).

\begin{tabular}{|l|l|l|}
\hline Fase & Valor experimental & Valor referencia \\
\hline & $2.813 \mathrm{~ms}$ & $800 \mathrm{~ms}-20.000 \mathrm{~ms}^{(6)}$ \\
Agua & $238 \mathrm{~ms}$ & $200 \mathrm{~ms}-750 \mathrm{~ms}^{(6)}$ \\
Lípidos & $951 \mathrm{~ms}$ & $950 \mathrm{~ms}-1.820 \mathrm{~ms}^{(6)}$ \\
Músculo & \multicolumn{2}{|}{} \\
\hline
\end{tabular}

Tabla IV. Valores T2 obtenidos a partir de la fórmula (B).

\begin{tabular}{|l|l|l|}
\hline Fase & $\begin{array}{l}\text { Valor } \\
\text { experimental }\end{array}$ & $\begin{array}{l}\text { Valor } \\
\text { referencia }\end{array}$ \\
\hline Agua & $704 \mathrm{~ms}$ & $110 \mathrm{~ms}-2.000 \mathrm{~ms}^{(6)}$ \\
Lípidos & $81 \mathrm{~ms}$ & $53 \mathrm{~ms}-94 \mathrm{~ms}^{(6)}$ \\
Músculo & $71 \mathrm{~ms}$ & $20 \mathrm{~ms}-67 \mathrm{~ms}^{(6)}$ \\
& & \\
\hline
\end{tabular}

Se establece que la curva $T_{1}$ (magnetización longitudinal) aumenta en función del tiempo. El contraste $T_{1}$ se genera usando valores de TR y TE cortos, valores muy bajos de TR generan muy poca señal (Figura 3 ). Por otro lado la curva $T_{\text {, }}$ (magnetización transversal) disminuye en función del tiempo. El contraste $T_{2}$ se genera usando valores TR y TE largos, valores altos de TE generan poca señal (Figura 4).

Se comprueba que el proceso de decaimiento de la magnetización transversal es mucho más rápido que la recuperación de la magnetización longitudinal (el $T_{1}$ es más largo que el $T_{2}$ ), la magnetización transversal se recupera prácticamente por completo después de 4-5 veces transcurrido el tiempo $T_{1}$.

Por otro lado, se verifica que en un líquido, la señal decae mucho más lentamente de lo que sucedería en un sólido. En un líquido puro, $\mathrm{T}_{2}$ puede alcanzar una magnitud de varios segundos $\mathrm{T}_{2}$.

\section{Conclusiones}

Se establece la posibilidad real de realizar cálculos de tiempo $T_{1}$ y $T_{2}$ en la práctica clínica. $\mathrm{Su}$ utilidad está dada porque entrega un valor cuantificable y reproducible, que permite la comparación de un paciente con otro o consigo mismo en el tiempo. En el caso de nuestro experimento se determinan los valores $T_{1}$, que corresponden a $951 \mathrm{~ms}$ para el músculo, $238 \mathrm{~ms}$ para la grasa y $2.813 \mathrm{~ms}$ para el agua. Los valores $\mathrm{T}_{2}$, son $71 \mathrm{~ms}$ para el músculo, $81 \mathrm{~ms}$ para la grasa y 704 ms para el agua.

\section{Bibliografía}

1. Gili J. Introducción biofísica a la resonancia magnética 2002.

2. Runge V, Ritz W. The Physics of clinical MR taught through images 2005; 35-37.

3. Morkisz J, Aquilia M. Technical magnetic resonance imaging 1996; 3: 47-61.

4. Pursey H. A note on the Carr-Purcell method of measuring nuclear magnetic resonance Relaxation Times Proc. Phys. Soc. 1961; 78: 808-811.

5. Siemens. Magnets, spíns and resonances 1997; 3453.

6. MR-Technology Information Portal (www.mr-tip.com). 